Supporting Information

\title{
Compositionally Graded AlGaN Nanostructures: the Strain Distribution and X-ray Diffraction Reciprocal Space Mapping
}

\author{
H. Stanchu',2, M. Auf der Maur ${ }^{3}$, A.V. Kuchuk ${ }^{*, 2}$, Yu.I. Mazur², M. Sobanska ${ }^{4}$, \\ Z.R. Zytkiewicz ${ }^{4}$, S. Wu${ }^{1}$, Z. Wang*,1, G. Salamo ${ }^{2}$ \\ ${ }^{1}$ Institute of Fundamental and Frontier Sciences, University of Electronic Science and \\ Technology of China, Chengdu 610054, P.R. China \\ ${ }_{2}^{2}$ Institute for Nanoscience and Engineering, University of Arkansas, Fayetteville 72701, USA \\ ${ }^{3}$ Department of Electronic Engineering, University of Rome Tor Vergata, Via del Politecnico 1, \\ 00133 Rome, Italy
}

${ }^{4}$ Institute of Physics Polish Academy of Sciences, al. Lotnikow 32/46, 02-668 Warsaw, Poland 
Table S1. Material constants used for the Al composition and strain calculations ${ }^{1}$.

\begin{tabular}{llllllll}
\hline \hline & $\mathrm{a}(\mathrm{nm})$ & $\mathrm{c}(\mathrm{nm})$ & $\begin{array}{l}\mathrm{C} 11 \\
(\mathrm{GPa})\end{array}$ & $\begin{array}{l}\mathrm{C} 12 \\
(\mathrm{GPa})\end{array}$ & $\begin{array}{l}\mathrm{C} 13 \\
(\mathrm{GPa})\end{array}$ & $\begin{array}{l}\mathrm{C} 33 \\
(\mathrm{GPa})\end{array}$ & $\begin{array}{l}\mathrm{C} 44 \\
(\mathrm{GPa})\end{array}$ \\
\hline $\mathrm{AlN}$ & 0.31130 & 0.49816 & 396 & 137 & 108 & 373 & 116 \\
$\mathrm{GaN}$ & 0.31893 & 0.51851 & 367 & 135 & 103 & 405 & 95 \\
\hline \hline
\end{tabular}

${ }^{1}$ Wallis, D. J. et al. Measuring the composition of AlGaN layers in GaN based structures grown on $150 \mathrm{~mm}$ Si substrates using (205) reciprocal space maps. Semicond. Sci. Technol 28, 094006 (2013). 

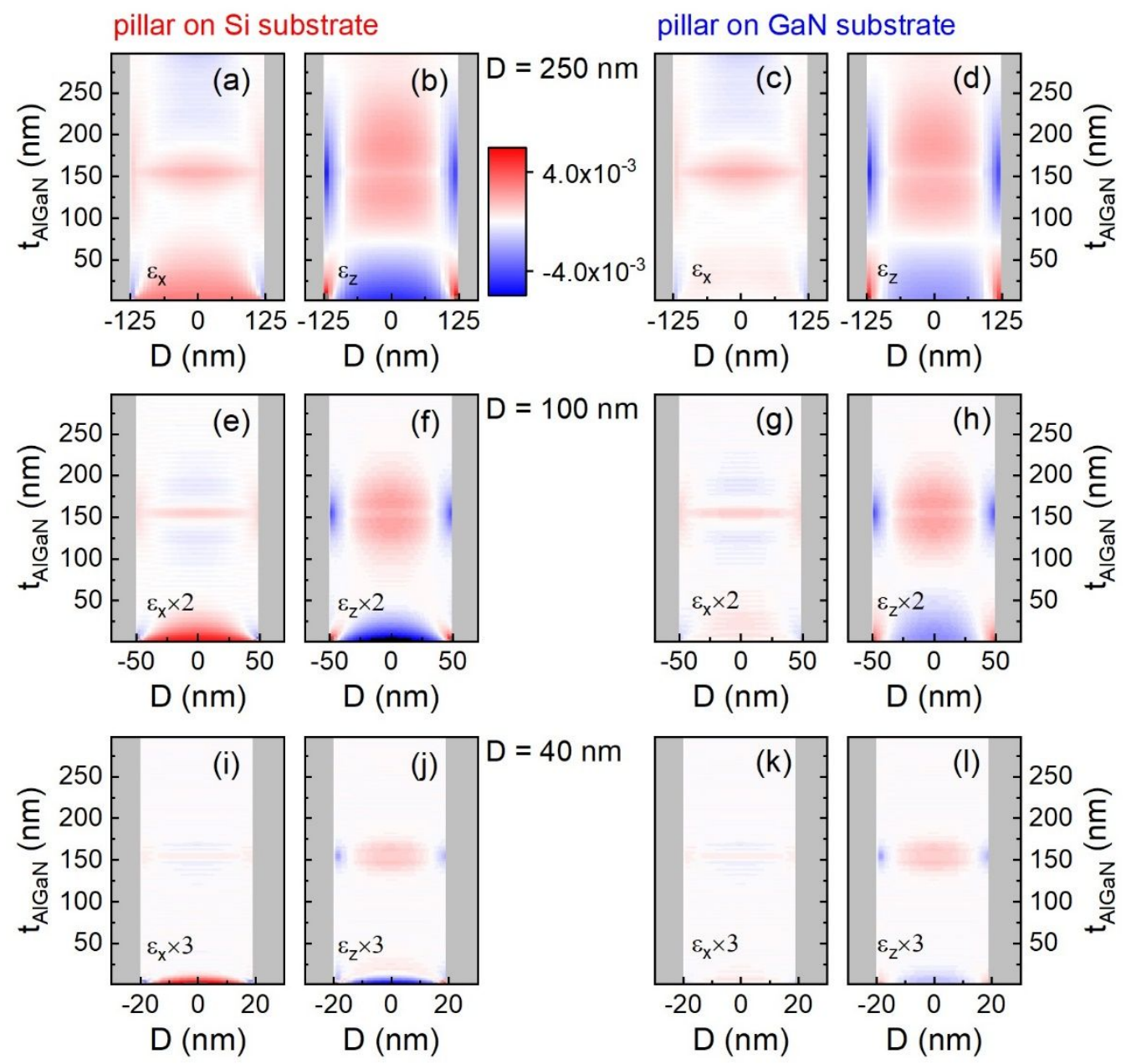

Figure S1. Calculated strain maps for compositionally graded $\mathrm{Al}_{\mathrm{x}} \mathrm{Ga}_{1-\mathrm{x}} \mathrm{N}$ pillars of different diameters on $\mathrm{Si}(111)$ and $\mathrm{GaN}(0001)$ substrates. For all the structures the $\mathrm{Al}$ composition profile is $\Lambda$-shaped with $x_{A l}^{\max }=50 \%$ (see Figure 4a). 